\title{
Indoor Positioning Algorithm of Wireless Sensor Based on RSSI Ranging Correction
}

\author{
Junjie Zhou \\ Shanghai Institute of Technology \\ Shanghai, China \\ zhoujunjiepk@126.com
}

\author{
Qinqin Shi \\ Shanghai Institute of Technology \\ Shanghai, China \\ shiqq@ sit.edu.cn
}

\begin{abstract}
With the growing popularity of the application of wireless sensor network, the demand to the accuracy of wireless network is increasingly strict, the traditional location algorithm has been difficult to fulfill the needs of practicable applications. This paper proposes a new RSSI positioning algorithm based on the triangle positioning algorithm in accordance with the character of received signal strength and by modifying the proportion between path length and path loss to improve the ranging accuracy, and then uses the triangle centroid algorithm to estimate the coordinate position. Though simulation experiment, it turned out that the accuracy of algorithm has improved compared with triangle centroid positioning algorithm on the basis of RSSI.
\end{abstract}

Keywords-wireless Sensor Network; RSSI; Triangle Centroid Algorithm; Ranging Model; Environmental Factor

\section{INTRODUCTION}

With the popularization and development of internet of things technology, the application of positiontechnology is becoming wider and wider, and the requirement of the position accuracy is higher. The application of traditional GPS positioning technology can be found everywhere in the life, however, GPS position technology consumes too much current and can hardly be used in poor GSM signal environment. Therefore, it can hardly realize in the real-time monitoring in indoor environment. The wireless network can make up for the shortcomings of GPS. When researchers are monitoring and tracking by wireless sensor node, the accuracy of sensor node location information will cause the effectiveness of the sensor to collect data[1]. Therefore, how to improve the positional accuracy is the key point.

Currently, sensor network localization can be divided into two main types: positioning algorithm based on distance and positioning algorithm irrelevant to distance. Locationbased positioning technology through inter-node point of measuring distance of angle information, using trilateration or maximum likelihood estimation method of position to calculate the node position. Irrelevant to distance method is not necessary distance and angle information can be realized only based on network connectivity and other information.

RSSI positioning technology is a kind of positioning algorithm with based on distance, it has advantages of easy to implement, low cost and has board prospects in application. Many scholars have done research on it, and put forward improved algorithm. For example, article 7 put forward a RSSI-similarity positioning algorithm[7], it is estimate the location of unknown node by establishing anchor node matrix manner, this method improved the positioning accuracy, and average error between 0.21$1.6(\mathrm{~m})$.Nonetheless with the increase number of node the calculate energy consumption changed a lot, so it is difficult to use in case of massive deployment of nodes. Paper 8 process a method of positioning, using TSK fuzzy neighboring anchor nodes weighted centroid algorithm[8], determining the fuzzy membership function, genetic algorithm to optimize fuzzy membership function to get the right value, improve positioning accuracy by weighting range accuracy. For RSSI-based wireless sensor networks vulnerable to interference and fluctuation, paper 9 improved indoor multidimensional scaling RSSI location algorithm[9], using the node signal strength to establish dissimilarity matrices and multidimensional scaling method to solve the node relative coordinates. According to the actual coordinates of the reference node coordinate conversion flat four-parameter model by using particle swarm optimization parameters to estimate position of unknown nodes.

In the wireless sensor network technology, the greater the density of anchor nodes, the higher the accuracy of the positioning is, otherwise the opposite[4]. Under the condition of indoor positioning, if more positioning anchor nodes are deployed, the signal interference between sensors will make the RSSI measured value bring more error. In that case, in order to acquire higher indoor positioning accuracy, less anchor nodes shall be deployed. This paper aims at the condition of indoor positioning, ameliorated the triangle centroid algorithm based on RSSI, brought in a method based on attenuation factor correction to improve ranging accuracy of RSSI. Compared with triangle centroid algorithm, the improved algorithm has an increased accuracy of ranging of RSSI as well as positioning.

\section{TRIANGLE CENTROID ALGORITHM BASED OF RSSI RANG}

\section{A. RSSI Ranging Model}

In the wireless sensor network, researchers need to provide necessary number of locations as reference points, which called the anchor nodes. Number and location of 
anchor node are the main factors affecting the positioning accuracy, but increase the number of anchor node in the position system can't meet the needs of different environments. By improving network deployment and positioning algorithms can solve problems WSM positioning itself. But each system and algorithms are used to solve different problems or support different applications, which form the physical phenomena for positioning, networks, and many aspects of the energy demand, infrastructure and the complexity of time or space are different. In the indoor positioning environment, it difficult to deploy a miraculous number of anchor nodes, choice fewer nodes required RSSI location algorithm is more appropriate.

Positioning technology based on RSSI is a kind of technology which transmitted signal by anchor nodes, and the signal transmission loss is figured out according to the signal strength received by unknown contacts. The transmission loss is transformed in to range value by combining with the theoretical model, consequently, the distance between anchor nodes and unknowns nodes is obtained[6]. The RSSI-ranging model always uses the distribution model of logarithm-normality, as shown in (1).

$$
\mathrm{p}(\mathrm{d})[\mathrm{dBm}]=\mathrm{p}\left(\mathrm{d}_{0}\right)[\mathrm{dBm}]-10 * \mathrm{~N} * \lg \left(\frac{\mathrm{d}}{\mathrm{d}_{0}}\right)+\mathrm{X}(1)
$$

Among this formula, $\mathrm{p}(\mathrm{d})$ expressed as the signal strength that unknown nodes received form anchor nodes in the distance of $\mathrm{d}$. And $\mathrm{p}(\mathrm{d} 0)$ expressed as the strength of deamplification signal when the nodes reference range is usually takes $1(\mathrm{~m}) ; \mathrm{N}$ means scale factor between path length and path loss[2], it is environment-related and the general ranges is $2 \sim 4$. $\mathrm{X}$ is Gaussian random quantity with average amount of 0 .

In the actual ranging, researchers always use $1(\mathrm{~m}), \mathrm{X}=$ $0(\mathrm{dBm})$ to simplify RSSI logarithm-normality model, as the (2) shows.

$$
\mathrm{p}(\mathrm{d})=\mathrm{p}-10 * \mathrm{~N} * \lg (\mathrm{d})
$$

Equation $(2)$ can be translated into $d=10^{\wedge(p-p(d)) /(10 * N)}, d$ is the distance measured between nodes. In practice, $p$ changes small, the accuracy of variable $\mathrm{N}$ determines the accuracy of the distance measurement.

\section{B. Trangle centroid algorithm}

After got the distance between anchor node and unknown node, researchers need to calculate the position of unknown node by positioning algorithm. Currently there are some modes like triangle centroid location algorithm, trilateration mode, triangulation mode, maximum likelihood estimation, minimax location algorithm. In the indoor positioning environment, choice triangle centroid location algorithm, it can reduce the impact of error of ranging by the geometric principle, and only need three anchors to locate.

Triangle centroid algorithm[3], as Figure 1 shown, anchor nodes A, B, C whose coordinates are (xa, ya), (xb , $\mathrm{yb})$ and $(\mathrm{xc}, \mathrm{yc})$. Researchers can measure the distance between anchor nodes and unknown node D ( $\mathrm{x}, \mathrm{y})$ by RSSI model.

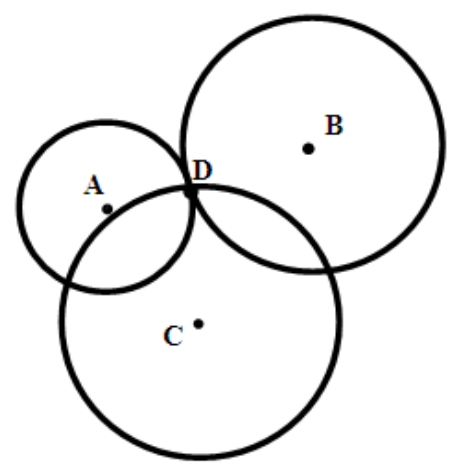

Figure 1. Trangle centroid algorithm

In the absence of the error environment, measured distance between anchor node and unknown is the actual value between them. Use $\mathrm{A}, \mathrm{B}, \mathrm{C}$ respectively as the center of circle and use the distance of anchors to unknown nodes as radius to draw circles. Three circular intersections at one point, this point $\mathrm{D}$ is the location of unknown node, researchers can use trilateration mode to estimate the position.

However, the signal strength which measured during the actual measurement may have errors due to the influence of complex environment and some other factors[5].For example, multipath attenuation and non-of-sight blockage will both affect the value of RSSI, so the measured distance is greater than the actual distance between two points. If researchers use A, B, C respectively as the center of circles and use the distance of anchors to unknown nodes as radius to draw circle, the three rounds can't intersect at one point, in the Figure 2. There will be two points of intersection between each two circles, choice the intersection point which closer to the third center point for calculating. And researchers will get three points E, F, G. Three points in a triangle, the centroid D' of centroid triangle is the estimated position of unknown nodes.

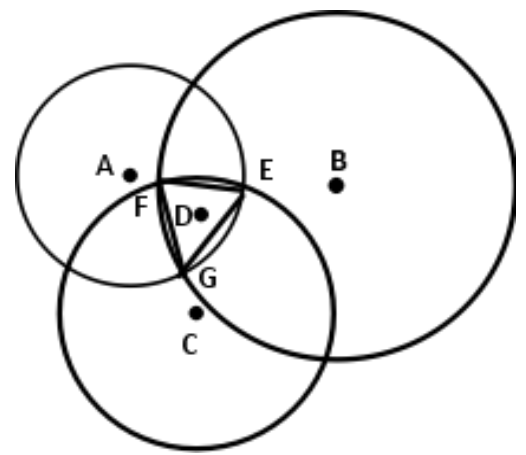

Figure 2. Trangle centroid algorithm in Actual

According to (3), researchers can draw the coordinate (xe, ye) of $E$, the coordinate of $F$ and $G$ can be drawn out in a similar way. Thus the estimate of centroid of triangle is the location of direct point $D^{\prime}=\left(\frac{x_{e}+x_{f}+x_{g}}{3}, \frac{y_{e}+y_{f}+y_{g}}{3}\right)$. 


$$
\left\{\begin{array}{l}
\sqrt{\left(x_{e}-x_{a}\right)^{2}+\left(y_{e}-y_{a}\right)^{2}} \leq R_{a} \\
\sqrt{\left(x_{e}-x_{b}\right)^{2}+\left(y_{e}-y_{b}\right)^{2}}=R_{b} \\
\sqrt{\left(x_{e}-x_{c}\right)^{2}+\left(y_{e}-y_{c}\right)^{2}}=R_{c}
\end{array}\right.
$$

\section{IMPROVED METHOD OF POSITIONING ALGORITHM}

\section{A. Correction of RSSI ranging attention factor}

When using the triangle centroid algorithm to position, the higher accuracy of the distance between nodes measured by RSSI, the more accurate the position result will be. In the actual environment, signal is easily affected by reflex, diffraction, none-of-sight and some other factors during transmission. Since the anchor node and unknown node between the closer, the greater impact on the unknown node and the environment factor is more accurate[10]. In that case, the signal strength measured by node will change, thus the distance of RSSI ranging will be affected, and finally large error of the position result will be brought as a result.

As the environment changes, proportion between path length and path loss also changed and these changes will lead to the value of RSSI error. Therefore, researchers can improve ranging accuracy of RSSI by correcting attenuation factor in RSSI model, thus the triangle centroid accuracy of positioning can be improved. During positioning, the distance between the anchor nodes is not changed, but RSSI measurement distance between the anchor nodes will change because of impact on the environment. According to the actual distance and estimate distance between anchor nodes $\mathrm{A}, \mathrm{B}$ and $\mathrm{C}$ to carry out attenuation factors between them, in this way to correct the attenuation factor during actual measuring.

The distance between anchor A and B, B and C, A and C are respectively $\mathrm{d} 1, \mathrm{~d} 2$ and $\mathrm{d} 3$; the corresponding RSSI value are $\mathrm{p} 1, \mathrm{p} 2$ and $\mathrm{p} 3$. According to (2), researchers can form a equation set (4).

$$
\left\{\begin{array}{l}
\mathrm{f}\left(\mathrm{x}_{1}\right)=\mathrm{p}-\mathrm{p}_{1}-10 * \mathrm{n}_{1} * \lg \left(\mathrm{d}_{1}\right) \\
\mathrm{f}\left(\mathrm{x}_{2}\right)=\mathrm{p}-\mathrm{p}_{2}-10 * \mathrm{n}_{2} * \lg \left(\mathrm{d}_{2}\right)(4) \\
\mathrm{f}\left(\mathrm{x}_{3}\right)=\mathrm{p}-\mathrm{p}_{3}-10 * \mathrm{n}_{3} * \lg \left(\mathrm{d}_{3}\right)
\end{array}\right.
$$

If put forward a hypothesis that $f(x 1)=f(x 2)=0$, researchers can obtain the value of $\mathrm{n} 1$ and $\mathrm{n} 2$, and then $\mathrm{n} \cdot 3=$ $(\mathrm{n} 1+\mathrm{n} 2) / 2$, use n' 3 tocalculatef' $(\mathrm{x} 3)$. In the same way, calculate n'1 andn'2, $f^{\prime}(x 3)$ and $f^{\prime}(x 3)$. Chose the minimum value of $\left|f^{\prime}(x n)\right|$, then the corresponding value of n'n is the RSSI environmental factors to correct the distance between nodes with unknown nodes.

\section{B. Flow of Positioning Algorithm}

Figure 3 is the flow chart of improved method of positioning algorithm. Compare with the triangle centroid algorithm, the improved algorithm can get better accuracy of ranging by correcting environmental factors. And the process of algorithm is as follows:

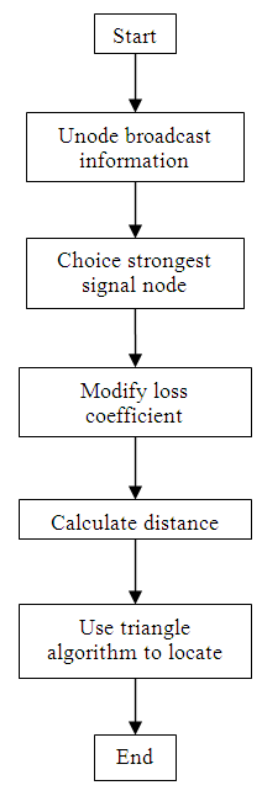

Figure 3. Flowchart of positioning algorithm

- Anchor nodes broadcast location information of itself such as coordinate and ID number, and then unknown nodes receive the signal strength information of each anchor node.

- During the RSSI ranging, the shorter distance has more accuracy of ranging. After unknown nodes receiving anchor nodes information, the information will be sorted according to the RSSI form strong to weak.

- Created a collection of anchor nodes:

- $\quad$ Bnode $=\{a 1, a 2, a 3, a 4\}$

- Collection of anchor nodes coordinates:

- $\quad$ Unode $=\{(\mathrm{x} 1, \mathrm{y} 1),(\mathrm{x} 2, \mathrm{y} 2),(\mathrm{x} 3, \mathrm{y} 3),(\mathrm{x} 4, \mathrm{y} 4)\}$

- Collection of anchor nodes signal strength:

- $\quad \mathrm{RSSI}=\{\mathrm{R} 1, \mathrm{R} 2, \mathrm{R} 3, \mathrm{R} 4\}$

- Choice three the strongest signal strength of the anchor nodes to calculate the position of unknown nodes. The path loss coefficient $\mathrm{N}$ of different anchor is measured out through the actual distance of any two RSSI values of the anchor nodes.

- To measure the distance between anchor nodes and unknown nodes by using path loss coefficient, in that case, the corrected distance between unknown nodes and anchor nodes will be carried out.

- Using the triangle centroid location algorithm, and the correct distance between anchor nodes and unknown node to estimate position of the unknown node.

\section{ANALYSIS OF SIMULATION RESULT}

We can simulate ranging optimization algorithm by using Matlab7.0, and compare with the triangle centroid algorithm. The simulation is carrying out indoor of $10^{*} 10(\mathrm{~m} 2)$ square; take the four nodes of the square as anchor nodes coordinate $(0,0),(0,10),(10,0),(10,10)$. When doing simulation, the unknown nodes shall be distributed randomly in the area of 
anchor nodes, and then compare the positioning accuracy between triangle centroid algorithm and optimization algorithm.

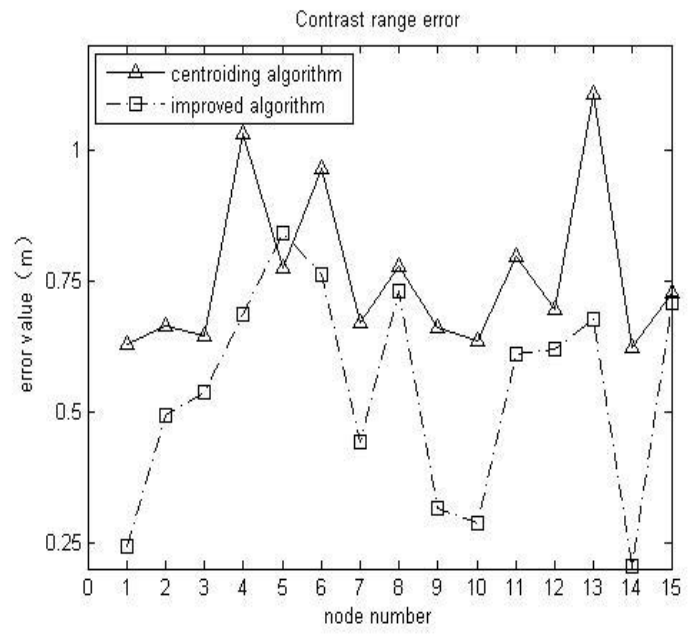

Figure 4. Contrast ranging error of different al gorithm

Mainly due to the impact location algorithm accuracy is the error of ranging between anchor node and unknown node. The Improve algorithm can correct environmental variable by measuring the actual distance between the anchor nodes, and ultimately improve the positioning accuracy. Figure 4 is a comparison of the two algorithms errors of ranging, from this picture researchers can clearly see the error of ranging improved a lot compared to the traditional method has been significantly improved. Experiments show that by modifying environmental factor to improve the range accuracy is feasible.

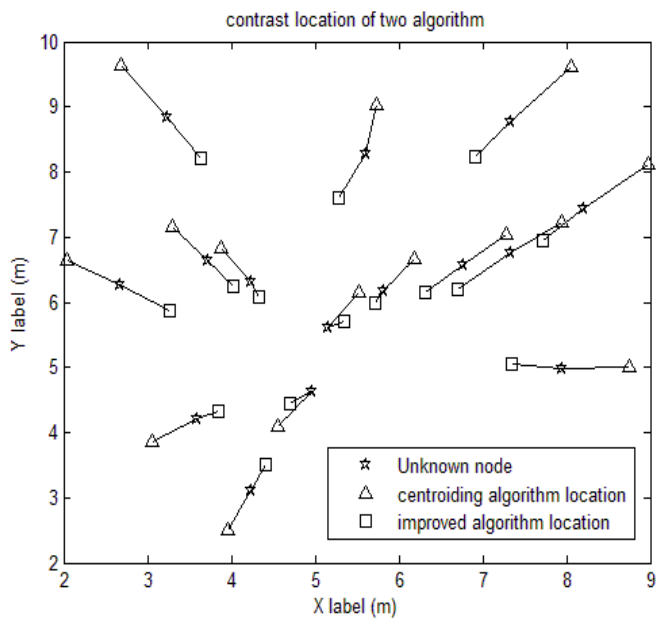

Figure 5. Contrast location of different algorithm

Figure 5 is the comparison of 15 unknown nodes of location results between triangle centroid algorithms and improved positioning algorithm. In simulation, 15 unknown nodes are generated, the star is positions of unknown node; the triangle represented estimated positions of unknown nodes carried out by centroid positioning algorithms; the square means estimated positions of unknown node obtained by improved position algorithm; the square means estimated position of unknown nodes obtained by improved positioning algorithm. The figure shows that the estimated position of improved algorithm is closer to position of unknown nodes than centroid positioning algorithm.

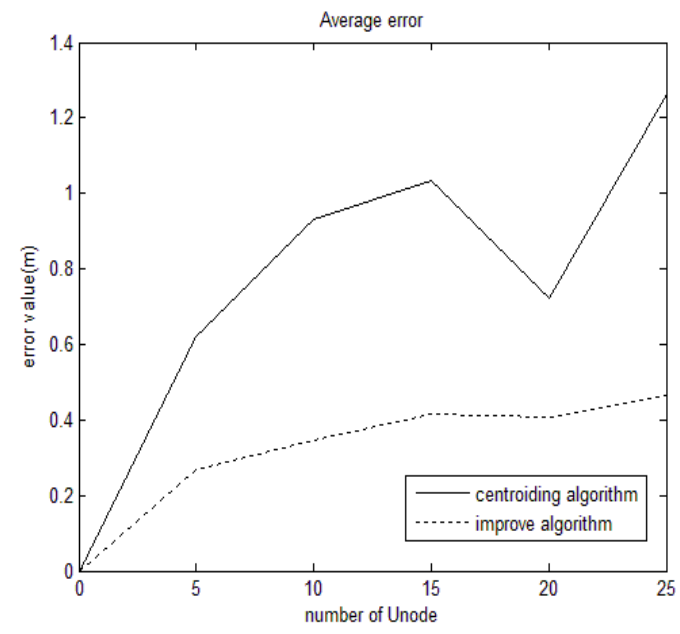

Figure 6. Contrast average error value

Figure 6 is comparison of error values between centroid algorithm and improved positioning algorithm. With the increase number of unknown nodes, the average error of centroid algorithm changed a lot, but the new algorithm error variance is small and the error curve tends to flatten. Improved algorithm has increased the accuracy and stability of triangle centroid positioning algorithm.

Simulation results show that the improved algorithm has better improvement in accuracy of positioning and better stability, because the current work is primarily made through the simulation, lack of hardware measurement data to support. Therefore, the next research will focus on algorithm performance in real environment, detection of possible energy loss algorithm, positioning time and other issues, and solve these problems. Eventually, implement the improved localization algorithm in practice.

\section{CONCLUSIONS}

In order to solve the problem of position errors produced by environment change, this paper presented the improved positioning algorithm based on triangle positioning algorithm, and uses the method of correction introducing environmental factor, in order to correct the distance between nodes during positioning and improve the positioning accuracy. Simulation test demonstrates that improved method has higher accuracy of positioning than the triangle centroid algorithm.

\section{REFERENCES}

[1] Capkun S, Hamdi M and Hubaux JP.GPS-Free Positioning in Mobile Ad-Hoc Networks[C]. Proceedings of the 34th Annual Hawaii Iternation Conference on System Science, Haiwaii, USA: IEEE Computing society, 2001. pp. 3481-3490. 
[2] Dongyong YANG, Dongyuan GU, Xiaojie FU. An Indoor Location Algorithm Base on RSSI-Similarity Degree[J]. Chinese Journal Of Sensors And Actuators, 2009, vol.22, pp.264-268.

[3] Sukhyun Y, Jaehum L, Wooyong C, Euntai K. Centroid Localization Method in Wireless Sensor Networks using TSK Fuzzy Modeling[C].Proceeding of 8th International Symposium on Advanced Intelligent System(ISIS2007). Sokcho City, Korea, 2007:971.

[4] Xin SHI, Aimin YIN, Xi CHEN. RSSI and Multidimensional Scaling Based Indoor Localization Algorithm[J]. Chinese Jounal of Scientific Instrument, 2012, vol.35, pp.261-267

[5] Jing LIU. A Study of Centroid Localization Algorithm and Based on Mobile Anchor Node Localization Algorithm in WSM. Zhengzhou: Zhengzhou University, 2012.
[6] Zepeng ZHU, Shuqiu YE. Weithted Centered Optimization Algorithm Based on RSSI[J]. Computer and Modernization, 2014, vol. 4, pp. 105-108.

[7] Iming SUN, Jianzhong LI, Yu CHEN,etc. Wireless Sensor Network. BeiJing[M]. Tsinghua University Press, 2005.

[8] Wei LIN, Chuangfeng CHEN. RSSI-based Triangle and Centroid Location in wireless Sensor Network[J]. Modern Electronic Technology. 2009, vol. 22, pp. 180-182.

[9] An optimized algorithm of triangle centroid localization based on RSSI ranging[C]. 2011 International Conference on Information, Services and Management Engineering[s.1]. IEEE, 2011, pp. 14581461.

[10] Rui GUO, Yinfei MA. The Optimization Strategy of Trianglecentroid Location Algorithm Based on RSSI[J]. Microelectronics \& Computer, 2014, vol. 3, pp.111-114. 